\title{
CRESCIMENTO E QUALIDADE DE MUDAS DE Eucalyptus urophylla X Eucalyptus grandis EM DIFERENTES RECIPIENTES
}

\author{
Ester Bühler Storck ${ }^{1}$, Lauri A mândio Schorn², Tatiele Anete Bergamo Fenilli ${ }^{2}$ \\ ${ }^{1}$ Bahia Specialty Cellulose Copener/Florestal, Camaçari, Bahia, Brasil - ester.storck@ hotmail.com \\ ${ }^{2}$ Universidade de Blumenau, Curto de Engenharia Florestal, Blumenau, Santa Catarina, Brasil - lauri.schorn@gmail.com; lschorn@furb.br; \\ tatifenilli@gmail.com; tfenilli@furb.br
}

Recebido para publicação: 10/12/2014 - Aceito para publicação: 27/11/2015

\begin{abstract}
Resumo
O experimento foi instalado com o objetivo de avaliar a qualidade e o desenvolvimento de mudas de Eucalyptus urophylla x Eucalyptus grandis em diferentes idades em viveiro e produzidas em diferentes volumes de tubetes de polipropileno, visando determinar a idade ótima de expedição das mudas para cada volume de recipiente estudado. O substrato utilizado foi uma mistura contendo esterco bovino, terra de subsolo e casca de arroz carbonizada, na proporção de 2:1:1. Foram utilizados tubetes de 55, 100 e $200 \mathrm{~cm}^{3}$. $\mathrm{O}$ delineamento experimental foi inteiramente casualizado, em esquema fatorial 3 (tubetes) x 5 (idades), com 15 tratamentos, cada um com 100 plantas, totalizando 1500 indivíduos. As plantas foram avaliadas em diferentes idades: 90, 120, 150, 180 e 210 dias. Foram mensuradas em cada muda as seguintes variáveis em cada idade: altura, diâmetro do colo, biomassa seca de raízes, biomassa seca aérea, biomassa seca total, número de raízes secundárias e índice de qualidade de Dickson. Realizou-se a análise de variância das variáveis, cujos resultados foram comparados pelo teste de Tukey a 5\% de probabilidade. Aos 90 dias, não se observaram diferenças significativas entre os tratamentos para a maioria das variáveis. Aos 180 dias houve diferenças significativas para todas as variáveis analisadas. Concluiu-se que a idade ótima para expedição de mudas em tubetes de $55 \mathrm{~cm}^{3}$ é aos 90 dias, aos 150 dias para mudas em tubetes de $100 \mathrm{~cm}^{3}$ e aos 180 dias para mudas em tubetes de $200 \mathrm{~cm}^{3}$.
\end{abstract}

Palavras-chave: Qualidade; mudas; biomassa seca; raízes.

\begin{abstract}
Growth and quality of Eucalyptus urophylla S.T. seedlings Blake X Eucalyptus grandis Hill (ex Maiden) in different containers. An experiment was carried out to study the quality and the development of Eucalyptus urophylla $\mathrm{x}$ Eucalyptus grandis seedlings at different ages in nursery and produced (grown) in different sized polypropylene tubes, aiming to determinate the optimum age and size tube to dispatch the seedlings to the field. A mixture of manure bovine, subsoil and carbonized rice husk, in the proportion of 2:1:1. It was utilized tubes of 55, 100 and $200 \mathrm{~cm}^{3}$. The experimental plan was entirely randomized design with 15 treatments, on factorial 3 (tubes) x 5 (age) and 100 plants each, totaling 1500 individuals. The plants were measured if different ages: 90, 120, 150, 180 and 210 days. The following variables was measured in each seedling and age: height, stem diameter, roots dry biomass, shoot dry biomass, total biomass, secondary roots number and Dickson quality index. Results were compared by the analysis of variance (ANOVA), and the averages were compared by Tukey's test in a 5\% probability. At 90 days of age, there were no significant differences between the treatments for most variables. At 180 days of age, there were significant differences for all analyzed variables. It was concluded that the optimum age to dispatch the seedlings produced in $55 \mathrm{~cm}^{3}$ tubes is 90 days of age, 150 days of age to seedlings produced in $100 \mathrm{~cm}^{3}$ tubes and 180 days of age to seedling produced in $200 \mathrm{~cm}^{3}$ tubes.

Keywords: Quality; seedlings; dry biomass; roots.
\end{abstract}

\section{INTRODUÇÃo}

O consumo crescente de produtos oriundos de reflorestamentos exige o aperfeiçoamento constante das técnicas silviculturais de produção, de forma que o atendimento dessas demandas seja verticalizado e não somente através da expansão de áreas reflorestadas. Dentro des se contexto está a produção de mudas florestais, etapa importante na produção florestal, que exige estudos que possibilitem a sua melhor eficiência.

Segundo Carneiro (1995), na fase de produção de mudas de qualquer espécie, sempre se deve almejar um padrão de qualidade que possibilite o melhor crescimento e a maior sobrevivência no plantio em campo. Várias práticas culturais contribuem para isso na fase de viveiro, podendo-se citar, entre outros, tipo de 
recipiente, manejo da irrigação, fertilização, sombreamento, micorrização, podas, aclimatação, seleção, densidade de cultivo, transporte e armazenamento.

Nesse sentido, entre as diversas práticas culturais capazes de alterar a qualidade morfofisiológica das mudas está a escolha do tamanho dos recipientes. Diversos autores destacam a importância das dimensões dos recipientes (CARNEIRO, 1995; CALDEIRA et al., 2007; GOMES et al., 2013), ressaltando que o uso de recipientes maiores que os volumes recomendáveis resultam em custos desnecessários de materiais e que os volumes dos recipientes devem variar com as características de cada espécie e respectivo tempo de permanência no viveiro. Outros autores (BARROSO et al., 2000; FREITAS et al., 2006; FREITAS et al., 2013) observaram que mudas de eucalipto produzidas em recipientes de maior volu me apresentaram maior desenvolvimento inicial em campo, reduzindo gastos com controle de plantas daninhas.

$\mathrm{O}$ uso de recipientes inadequados, com volumes menores que o necessário para determinados tamanhos de mudas, também é u ma das causas mais comuns da malformação do sistema radicular das mudas no viveiro, por promoverem distribuição anormal de raízes laterais e superficiais, ocasionando desequilíbrio na absorção de água e nutrientes em quantidades suficientes para atender às necessidades da planta (MAFIA et al., 2005). Isso resulta em um quadro sintomatológico típico de deficiência hídrica ou nutricional, em consequência do desequilíbrio entre raize parte aérea (ELOY, et al., 2013).

Já a persistência de deformações radiculares em função da restrição no viveiro pode reduzir, ou atrasar, o crescimento das plantas no campo (FREITAS et al., 2005), o que pode acarretar maio res custos na implantação florestal (MATTEI, 1993; FREITAS et al., 2005; NEVES et al., 2005). Esses autores destacam ainda a importância de priorizar metodologias de produção de mudas que não provoquem deformações em suas raízes.

Dessa forma, torna-se necessário avaliar e conhecer as relações entre tamanhos de recipientes e as idades limites das mudas para permanência em viveiro nesses recipientes sem que ocorra prejuízo no seu crescimento.

Eucalyptus urograndis é um híbrido desenvolvido no Brasil através de cruzamentos do E. urophylla e com E. grandis. O objetivo do cruzamento dessas duas espécies, segundo Carvalho (2000), foi obter plantas com um bom crescimento, característica do E. grandis, assim como com um leve aumento na densidade da madeira, além de melhorias no rendimento e propriedades físicas da celulose, características do E. urophylla. Além de melhores propriedades da madeira, E. urograndis apresenta boas características quanto à adaptação aos diferentes sítios florestais e elevada resistência ao fungo Puccina psiidi (MONTANA RI, 2007).

$\mathrm{O}$ objetivo desta pesquisa foi avaliar a qualidade de mudas do híbrido Eucalyptus urophylla $\mathrm{x}$ Eucalyptus grandis S.T. Blake em diferentes idades em viveiro e produzidas em diferentes volumes de tubetes de polipropileno, visando determinar a idade ótima de expedição das mudas para cada volume de recipiente estudado.

\section{MATERIAL E MÉTODOS}

O projeto foi desenvolvido no laboratório de silvicultura da Universidade Regional de Blumenau. O clima na região de Blumenau, segundo a classificação climática de Thomthwaite, é do tipo $\mathrm{B}_{2} \mathrm{~B}_{3}$ ra - Úmido Mesotérmico, sem deficiência de chuva em qualquer estação. Ocasionalmente são registradas quedas de temperatura pouco abaixo de $0{ }^{\circ} \mathrm{C}$ nos meses de junho, julho e agosto, dependendo da intensidade da passagem das massas de ar polar. As temperaturas médias anuais são atenuadas pela proximidade do mar. A precipitação anual varia entre 1.400 e $1.800 \mathrm{~mm}$, distribuídos entre 120 e 140 dias de chuva durante o ano, com umidade relativa média entre 75 e $80 \%$ (SANTOS, 1996).

Para o desenvolvimento do estudo, foi utilizado o híbrido Eucalyptus urophylla x Eucalyptus grandis, cujas sementes foram adquiridas no Instituto de Pesquisas e Estudos Florestais (IPEF) de São Paulo.

Foram implantados quinze tratamentos, caracterizados por volumes de tubetes e idades de mudas em viveiro. Os volumes de tubetes utilizados foram de $55 \mathrm{~cm}^{3}, 100 \mathrm{~cm}^{3}$ e $200 \mathrm{~cm}^{3}$. Esses tubetes apresentavam dimensões de $3,0 \mathrm{~cm} \times 12,0 \mathrm{~cm}$ x $1,0 \mathrm{~cm}, 4,0 \mathrm{~cm} \mathrm{x} 14 \mathrm{~cm} \mathrm{x} 1,5 \mathrm{~cm}$ e $6,0 \mathrm{~cm}$ x $18,0 \mathrm{~cm} \mathrm{x} \mathrm{2,0} \mathrm{cm,} \mathrm{para} \mathrm{diâmetro}$ superior, altura e diâmetro inferior, respectivamente. Todos apresentavam forma cilíndrica e continham seis estrias internas.

Para todos os tratamentos, foi utilizado substrato contendo esterco bovino, solo de horizonte B e casca de arroz carbonizada, na proporção de 2:1:1. O delineamento experimental foi inteiramente casualizado, em esquema fatorial 3 x 5, com quinze tratamentos e cinco repetições por tratamento. Cada parcela era co mposta por vinte plantas, acondicionadas individualmente nos recipientes. Foram necessárias 100 plantas por tratamento, totalizando 1.500 plantas. O experimento foi instalado em setembro de 2012 e concluído em abril de 2013.

Foram retiradas aleatoriamente quatro plantas de cada parcela em cada ocasião de avaliação. Foram mensuradas em cada planta e calculadas para cada parcela as seguintes variáveis: altura $(\mathrm{H})$, diâmetro do colo 
(DC), biomassa seca de raízes (BSR), bio massa seca aérea (BSA), bio massa seca total (BST), núme ro de raízes secundárias e índice de qualidade de Dickson (IQD). Para obtenção de biomassa seca, as plantas foram submetidas a secagem em estufa a $70^{\circ} \mathrm{C}$ até a obtenção de peso constante, procedimento que levou aproximadamente três dias. O número de raízes secundárias foi obtido após a retirada do substrato e lavação das raízes em água corrente, pela contagem de raízes formadas a partir da raiz principal.

As avaliações das variáveis biométricas e de biomassa foram realizadas aos 90, 120, 150, 180 e 210 dias. Para cada variável mensurada, obtiveram-se os incrementos médios mensais. Aos 90 dias, foram obtidos os incrementos médios mensais para cada variável através da divisão dos valores mensurados nessa ocasião pela respectiva idade (três meses). Para as demais ocasiões de avaliação (120, 150, 180 e 210 dias), obtiveram-se os incrementos periódicos mensais, subtraindo-se os valores médios obtidos em cada ocasião das médias obtidas na ocasião anterior.

Para a comparação dos tratamentos, inicialmente os dados foram submetidos ao teste de Bartlett para verificação da homogeneidade das variâncias e posteriormente foi realizada a análise da variância para os incrementos mensais de todas as variáveis analisadas. As médias foram comparadas pelo teste de Tukey, utilizando-se o nível de probabilidade de 5\%. Foi realizada a análise da regressão para os três tamanhos de tubetes utilizados, com os incrementos em bio massa seca total (variável dependente) obtidos mensalmente em cada idade (variável independente). Através da análise da regressão, selecionou-se o modelo que apresentou melhor ajuste aos dados através do maior valor de coeficiente de determinação $\left(\mathrm{R}^{2}\right)$.

Para se verificar a relação entre as variáveis utilizadas na pesquisa, foi realizada uma análise de agrupamentos, utilizando-se as correlações entre as variáveis e através do método de ligação de simples.

As análises de homogeneidade da variância, análise da variância e teste de Tukey foram realizados através do software Assistat versão 7.7 beta, enquanto as análises da regressão e de agrupamentos foram obtidas através do software PAST.

\section{RES ULTADOS E DISCUSSÃO}

Todas as variáveis analisadas apresentaram dados com variâncias considerados homogêneas $(p<0,05)$, conforme resultados obtidos através do teste de Bartlett.

Houve diferenças significativas no crescimento das mudas de Eucalyptus urophylla x Eucalyptus grandis entre as idades em viveiro e entre o tamanho dos tubetes, bem como para a interação entre os dois fatores. Para todos os parâmetros, obtiveram-se maiores valores em tubetes de maiores volumes. Es se resultado foi o esperado, uma vez que o tubete de maior volume $\left(200 \mathrm{~cm}^{3}\right)$ ultrapassa quatro vezes o menor $\left(55 \mathrm{~cm}^{3}\right)$ e proporciona menor restrição ao desenvolvimento das mudas (Tabela 1).

Os incrementos em altura, diâmetro do colo, biomassa seca aérea, biomassa seca total, número de raízes secundárias e índice de qualidade de Dickson das mudas foram crescentes e significativamente diferentes com os tamanhos de tubetes a partir de 90 dias. Nessa idade, as mudas produzidas em tubetes de $55 \mathrm{~cm}^{3}$ apresentaram, com exceção de bio massa seca de raízes, médias das variáveis significativamente inferiores quando comparadas com mudas em tubetes de maiores volumes. Esse fato mostra que aos 90 dias houve restrição ao desenvolvimento das mudas, ocasionada pelo volume de substrato no tubete. A biomassa seca de raízes mostrou diferenças significativas entre volumes de tubetes somente a partir de 120 dias.

Os incrementos máximos para as variáveis analisadas foram obtidos em idades distintas. Para $\mathrm{H}$, os incrementos máximos foram obtidos aos 180 dias para as mudas produzidas em tubetes de 55 e $100 \mathrm{~cm}^{3}$. Para DC e BSR, os incrementos máximos foram obtidos a partir de 150 dias em tubetes de $55 \mathrm{~cm}^{3}$, e a partir de 180 dias para tubetes de $100 \mathrm{~cm}^{3}$. Já para BSA, ocorreu estagnação dos incrementos já aos 90 dias em tubetes de $55 \mathrm{~cm}^{3}$ e aos 180 dias e m tubetes de $100 \mathrm{~cm}^{3}$, enquanto que para MST os valores máximos foram observados aos 150 e 180 dias para tubetes de 55 e $100 \mathrm{~cm}^{3}$, respectivamente. O NRS apresentou valores variáveis entre as mudas produzidas nos três volumes até 150 dias e não foi possível detectar efeitos de tubetes sobre o NRS até essa idade. O IQD mostrou resultados semelhantes ao NRS, pois aos 150 dias as mudas em tubetes de 55 e de $100 \mathrm{~cm}^{3}$ apresentaram os valores máximos para essa variável. As mudas produzidas em tubetes de $200 \mathrm{~cm}^{3}$ apresentaram valores crescentes em quase todas as variáveis estudadas, sem evidenciar valores máximos aos 210 dias. So mente o NRS apresentou valores máximos entre 180 e 210 dias para esse volume de tubete (Tabela 1).

Os resultados para alturas das mudas são convergentes com aqueles obtidos por Freitas et al. (2013), que verificaram maior incremento em altura em tubete de maior volume para E. urophylla, E. robusta e Corymbia citriodora. No entanto, os incrementos máximos em altura no presente trabalho foram verificados em tubetes maiores e em idades mais tardias, diferenciando-se nesse aspecto dos resultados de Freitas et al. (2013), que obtiveram os máximos incrementos em altura em tubete de maior volu me, porém em idade mais precoce.

FLOREST A, Curitiba, PR, v. 46, n. 1, p. 39 - 46, jan. / mar. 2016.

Schorn, L. A. et al.

ISSN eletrônico 1982-4688

DOI: $10.5380 /$ rf.v46il .38907 
Tabela 1. Médias dos tratamentos das variáveis altura (H), diâmetro do colo (DC), biomassa seca radicular (BSR), bio massa seca aérea (BSA), bio massa seca total (BST), número de raízes secundárias (NRS) e índice de qualidade de Dickson (IQD) de mudas de Eucalyptus urophylla x Eucalyptus grandis através da interação dos fatores tamanhos de tubete e idades.

Table 1. Treatment means of variables, height (H), stem diameter (DC), root dry biomass (BSR), air dry biomass (BSA), total dry biomass (BST), number of secondary roots (NRS) and Dickson quality index (IQD) of Eucalyptus urophylla x Eucalyptus grandis seedlings through interaction of factors tubes sizes and ages.

\begin{tabular}{|c|c|c|c|c|c|c|c|c|c|c|}
\hline \multirow{2}{*}{$\begin{array}{l}\text { Variáveis } \\
\text { biométricas }\end{array}$} & \multirow{2}{*}{$\begin{array}{l}\text { Volumes de } \\
\text { tube te }\left(\mathrm{cm}^{3}\right)\end{array}$} & \multicolumn{9}{|c|}{ Idades } \\
\hline & & 90 & & 120 & & 150 & & 180 & & 210 \\
\hline \multirow{3}{*}{$\mathrm{H}(\mathrm{cm})$} & 55 & 12,20 & $\mathrm{bC}$ & 14,29 & $\mathrm{cBC}$ & 16,39 & $\mathrm{cB}$ & 21,86 & $\mathrm{cA}$ & $16,43 \mathrm{cB}$ \\
\hline & 100 & 15,52 & $\mathrm{aE}$ & 19,21 & $\mathrm{bD}$ & 22,89 & $\mathrm{bC}$ & 33,47 & $\mathrm{bA}$ & 29,03 bB \\
\hline & 200 & 14,81 & $\mathrm{aE}$ & 26,09 & $\mathrm{aD}$ & 37,37 & $\mathrm{aC}$ & 48,45 & $\mathrm{aB}$ & 58,00 aA \\
\hline \multirow{3}{*}{$\mathrm{DC}(\mathrm{cm})$} & 55 & 0,09 & $\mathrm{bC}$ & 0,11 & $\mathrm{cB}$ & 0,13 & $\mathrm{cA}$ & 0,14 & $\mathrm{cA}$ & $0,14 \mathrm{cA}$ \\
\hline & 100 & 0,12 & $\mathrm{aD}$ & 0,13 & $\mathrm{bC}$ & 0,15 & $b B$ & 0,18 & $\mathrm{bA}$ & 0,18 bA \\
\hline & 200 & 0,13 & $\mathrm{aE}$ & 0,18 & $\mathrm{aD}$ & 0,23 & $\mathrm{aC}$ & 0,27 & $\mathrm{aB}$ & 0,30 aA \\
\hline \multirow{3}{*}{ BSR (g) } & 55 & 0,09 & $\mathrm{aC}$ & 0,26 & $\mathrm{bB}$ & 0,44 & bA & 0,23 & $\mathrm{cB}$ & $0,21 \mathrm{cB}$ \\
\hline & 100 & 0,15 & $\mathrm{aD}$ & 0,27 & $\mathrm{bC}$ & 0,39 & $\mathrm{bAB}$ & 0,45 & $\mathrm{bA}$ & $0,32 \mathrm{bBC}$ \\
\hline & 200 & 0,14 & $\mathrm{aE}$ & 0,38 & $\mathrm{aD}$ & 0,61 & $\mathrm{aC}$ & 0,77 & $\mathrm{aB}$ & 1,04 aA \\
\hline \multirow{3}{*}{ BSA (g) } & 55 & 0,11 & $\mathrm{bA}$ & 0,13 & $\mathrm{cA}$ & 0,16 & $\mathrm{cA}$ & 0,20 & $\mathrm{cA}$ & $0,11 \mathrm{cA}$ \\
\hline & 100 & 0,19 & $\mathrm{abB}$ & 0,24 & $\mathrm{bB}$ & 0,29 & $b B$ & 0,45 & $\mathrm{bA}$ & 0,44 bA \\
\hline & 200 & 0,22 & $\mathrm{aE}$ & 0,54 & $\mathrm{aD}$ & 0,86 & $\mathrm{aC}$ & 1,26 & $\mathrm{aB}$ & $1,91 \mathrm{aA}$ \\
\hline \multirow{3}{*}{ BST (g) } & 55 & 0,20 & $\mathrm{bC}$ & 0,40 & $\mathrm{bB}$ & 0,59 & bA & 0,43 & $\mathrm{cAB}$ & $0,32 \mathrm{cBC}$ \\
\hline & 100 & 0,34 & $\mathrm{abD}$ & 0,51 & $\mathrm{bCD}$ & 0,68 & $\mathrm{bBC}$ & 0,90 & $\mathrm{bA}$ & $0,76 \mathrm{bAB}$ \\
\hline & 200 & 0,37 & $\mathrm{aE}$ & 0,92 & $\mathrm{aD}$ & 1,48 & $\mathrm{aC}$ & 2,03 & $\mathrm{aB}$ & 2,96 aA \\
\hline \multirow{3}{*}{ NRS } & 55 & 22,60 & $\mathrm{bD}$ & 31,20 & $\mathrm{bBC}$ & 39,20 & $\mathrm{abA}$ & 35,40 & $\mathrm{cAB}$ & $27,40 \mathrm{cCD}$ \\
\hline & 100 & 33,80 & $\mathrm{aB}$ & 34,80 & $a b A B$ & 35,80 & bAB & 41,60 & $\mathrm{bA}$ & 35,00 bAB \\
\hline & 200 & 34,00 & $\mathrm{aC}$ & 37,60 & $\mathrm{aBC}$ & 41,80 & $\mathrm{aB}$ & 51,60 & $\mathrm{aA}$ & 55,80 aA \\
\hline \multirow{3}{*}{ IQD } & 55 & 0,01 & $\mathrm{bC}$ & 0,03 & $\mathrm{bB}$ & 0,05 & bA & 0,03 & $\mathrm{cBC}$ & $0,03 \mathrm{cBC}$ \\
\hline & 100 & 0,02 & $\mathrm{abB}$ & 0,034 & $\mathrm{bAB}$ & 0,04 & bA & 0,04 & $\mathrm{bA}$ & 0,04 bA \\
\hline & 200 & 0,03 & $\mathrm{aE}$ & 0,06 & $\mathrm{aD}$ & 0,08 & $\mathrm{aC}$ & 0,10 & $\mathrm{aB}$ & 0,14 aA \\
\hline
\end{tabular}

As médias seguidas pela mesma letra maiúscula nas linhas e minúscula nas colunas não diferem estatisticamente entre si ao nível de $5 \%$ de probabilidade.

As alturas das mudas de Eucalyptus consideradas ideais para expedição têm sido entre 20 a $40 \mathrm{~cm}$ (ALFENAS et al., 2004), que são obtidas, em geral, com idade próxima de 90 dias e em tubetes de $55 \mathrm{~cm}^{3}$. Os resultados obtidos no presente trabalho mostraram que até 90 dias as mudas de E. urophylla x E. grandis atingiram alturas médias superiores a $20,0 \mathrm{~cm}$ em tubetes de $55 \mathrm{~cm}^{3}$, e alturas superiores a $40 \mathrm{~cm}$ quando em tubetes de $100 \mathrm{ou} 200 \mathrm{~cm}^{3}$, com médias de incremento mensal próximas de $15 \mathrm{~cm}$ (Tabela 01 ).

A altura é considerada um dos parâmetros mais antigos na classificação e seleção de mudas (PARVIA INEN, 1990) e ainda continua apresentando uma contribuição importante, podendo ser indicada como um parâmetro para essa avaliação. Ela expressa bem a qualidade das mudas, porém, segundo alguns autores, não deve ser analisada isoladamente, e sim com outros parâmetros, como diâmetro do colo, bio massa seca total e relação bio massa seca das raízes/biomassa seca da parte aérea (CARNEIRO, 1995; GOMES et al., 2003).

Outra variável analisada, o diâmetro do colo (DC), é considerada a mais adequada para indicar a capacidade de sobrevivência das mudas no campo (CARNEIRO, 1995; KNAPIK, 2005). O valor do DC considerado suficiente para expedição de mudas de Eucalyptus é $2 \mathrm{~mm}$ (CARNEIRO, 1995; ALFENAS et al., 2004). Os valores de incrementos mensais de DC obtidos para E. urophylla x E. grandis (Tabela 1) foram de $0,09 \mathrm{~cm}$ até aos 90 dias, resultando em valores médios de DC acumulado iguais ou superiores a $0,27 \mathrm{~cm}$ $(2,7 \mathrm{~mm})$ nessa idade. Os resultados evidenciam os efeitos dos dois fatores analisados sobre o diâmetro do colo 
de Eucalyptus urograndis. Gomes et al. (2003) obtiveram médias de DC em Eucalyptus grandis aos 90 e 120 dias bastante semelhantes às médias do presente trabalho, de forma semelhante ao que foi obtido por Freitas et al. (2013), em que maiores incrementos em DC foram verificados em mudas em tubetes de maiores volumes. Este resultado também sugere que o uso de tubetes com $200 \mathrm{~cm}^{3}$ permite a manutenção de mudas de E. urograndis emviveiro até 210 dias, no mínimo. Já o uso de tubetes com 55 e $100 \mathrm{~cm}^{3}$ permitem a manutenção das mudas em viveiro até 150 e 180 dias, respectivamente, sem pre juízo no crescimento em DC.

Os resultados para BSR indicam que em tubetes de 50 e $100 \mathrm{~cm}^{3}$ ocorreu restrição radicular a partir de 150 e 180 dias, respectivamente, fato não observado em mudas produzidas em tubetes de $200 \mathrm{~cm}^{3}$ e até a idade de 210 dias. A massa seca das raízes (MSR) é um dos mais importantes e melhores parâmetros para estimar a sobrevivência e o crescimento in icial das mudas (GOMES et al., 2013). Os resultados obtidos se assemelham aos encontrados em outros trabalhos, em que o tubete de menor volume causou restrição ao crescimento do sistema radicular (FREITAS et al., 2005; FREITAS et al., 2013; GOMES et al., 2013). Para BSA e BST, os resultados indicam que ocorreu restrição ao desenvolvimento em tubetes de $55 \mathrm{~cm}^{3}$ já aos 90 dias, e em tubetes de $100 \mathrm{~cm}^{3}$ aos 180 dias. Para as mudas produzidas em tubetes de $200 \mathrm{~cm}^{3}$ não houve restrição ao desenvolvimento da BSA e BST até 210 dias. Esse resultado sugere que a biomassa aérea manifesta os efeitos limitantes do menor volume de tubete mais precocemente em relação à biomassa de raízes. Os resultados também sugerem que os maiores valores de BSR, BSA e BST em tubetes de $200 \mathrm{~cm}^{3}$ estão relacionados ao maior volu me para o desenvolvimento das raízes e consequentemente da parte aérea (Figura 1). Trabalhos realizados com outros gêneros e espécies também apontaram resultados convergentes. Esse é o caso de Cunha et al. (2005), que, analisando o efeito das dimensões dos recipientes na qualidade de mudas de Tabebuia impetiginosa (Mart. ex DC.), concluíram que o desenvolvimento das plantas nos recipientes de maiores dimensões foi superior. De forma semelhante, Malavasi e Malavasi (2006) obtiveram melhor desempenho no desenvolvimento de mudas de Cordia trichotoma (Vell.) Arrab. ex Stend e de Jacaranda micrantha Cham. em tubetes com 120, 180 e $300 \mathrm{~cm}^{3}$ quando comparadas com mudas produzidas em tubetes de $55 \mathrm{~cm}^{3}$, após 180 dias do plantio a campo.

O recipiente de $55 \mathrm{~cm}^{3}$ tem sido o mais utilizado na produção de mudas de Eucalyptus. Entretanto, os resultados deste trabalho, assim como os resultados verificados por Freitas et al. (2013) apontam que recipiente de volume reduzido limita o desenvolvimento das espécies testadas em todas as características avaliadas, sendo importante as empresas de produção de mudas de eucalipto analisarem o uso de um recipiente de maior volu me, que poderá resultar na redução do ciclo de produção.
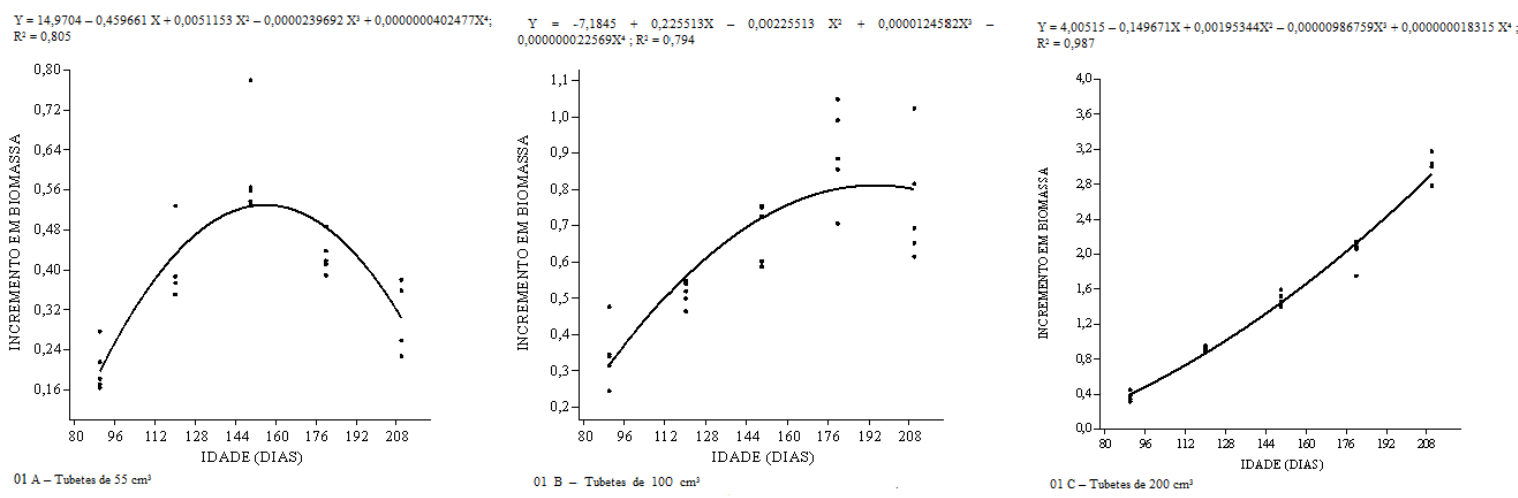

Figura 1. Incrementos em biomassa seca (g) de mudas de Eucalyptus urophylla x Eucalyptus grandis produzidas em três volumes de tubetes.

Figure 1. Dry biomass increments (g) of Eucalyptus urophylla x Eucalyptus grandis seedlings produced in three volumes of tubes.

O índice de qualidade de Dickson é considerado um bom indicador da qualidade das mudas, pois em seu cálculo são considerados a robustez e o equilíbrio da distribuição da biomassa na muda, ponderando os resultados de vários parâmetros importantes empregados para avaliação da qualidade (CALDEIRA et al., 2005; 2007). No entanto, a literatura evidencia que o IQD é u ma característica que pode variar em função da espécie, do manejo das mudas no viveiro, do tipo e proporção do substrato, do volume do recipiente e, principalmente, de acordo com a idade em que a muda foi avaliada (CALDEIRA et al., 2007; CALDEIRA et al., 2008a; CALDEIRA et al., 2008b; SAIDELLES et al., 2009; TRAZZI et al., 2010; GOMES et al., 2013).

FLOREST A, Curitiba, PR, v. 46, n. 1, p. 39 - 46, jan. / mar. 2016. 
Fonseca et al. (2002) relataram que as mudas com maiores valores de IQD apresentaram maiores valores de diâmetro do coleto, bio massa seca da parte aérea, do sistema radicular e total. No presente trabalho, os tratamentos com maiores valores de IQD também apresentaram elevados valores em BSR, BSA e BST, evidenciando uma forte correlação entre essas variáveis. Essa relação também foi evidenciada através da análise de agrupamento das variáveis (Figura 2), em que se observam menores distâncias entre IQD e as variáveis de biomassa (BSR, BAS e BST). Esse resultado é convergente com os dados obtidos por Go mes et al. (2003) em Eucalyptus grandis, com os parâmetros relacionados com os pesos de biomassa seca sendo os que mais contribuíram para a qualidade das mudas.

A análise das relações entre as variáveis também mostrou que $\mathrm{H}$ e DC apresentaram boa relação (Figura 2), e mbora com distâncias de IQD maiores em relação às variáveis de biomassa. No entanto, por serem variáveis de mensuração direta e não destrutivas, podem ser utilizadas para avaliar a qualidade das mudas de E. urophylla x E. grandis com razoável precisão, considerando os demais resultados obtidos. A BSA e a BST apresentaram a melhor relação (menor distância) com o IQD, seguidas de BSR, evidenciando a importância das variáveis de massa em avaliações do crescimento e da qualidade de mudas de E. urophylla x E grandis. O NRS apresentou a menor re lação como IQD e com as demais variáveis, mostrando ser menos eficiente para predizer o crescimento e a qualidade das mudas analisadas.

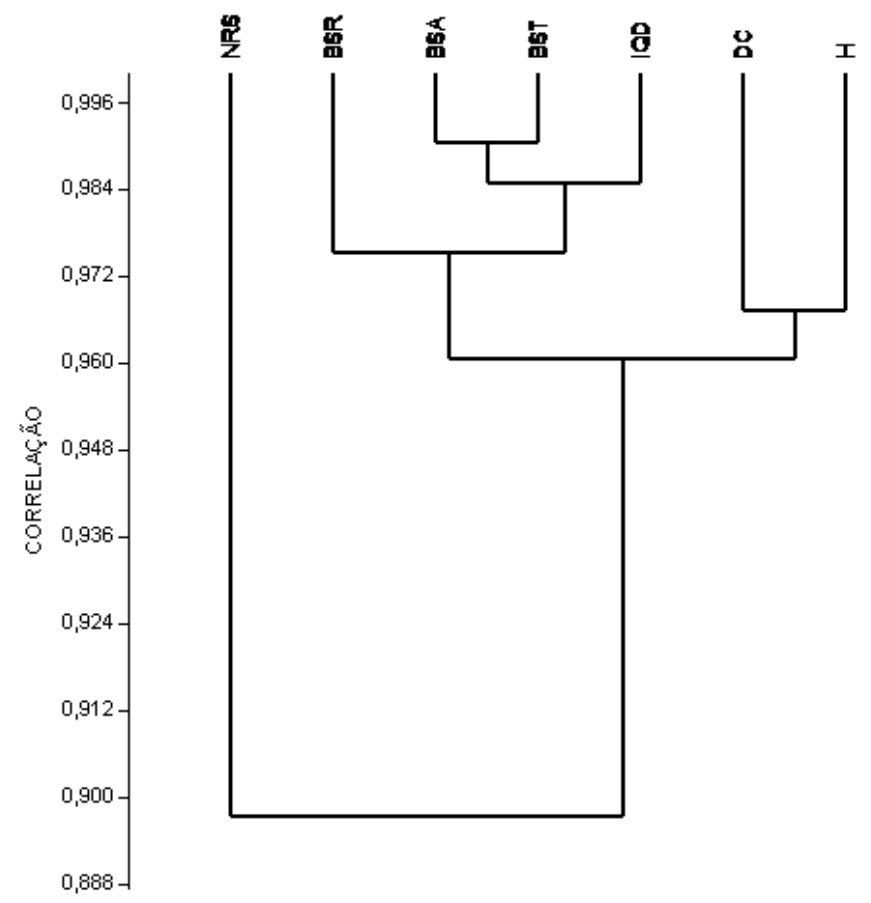

Figura 2. Análise de agrupamentos das variáveis utilizadas na avaliação das mudas de Eucalyptus urophylla $\mathrm{x}$ Eucalyptus grandis. $\mathrm{H}=$ altura; $\mathrm{DC}=$ diâmetro do colo; IQD = índice de qualidade de Dickson; BST = biomassa seca total; $\mathrm{BSA}=$ biomassa seca aérea; $\mathrm{BSR}=$ biomassa seca de raízes; NRS = nú mero de raízes secundárias

Figure 2. Cluster analysis of the variables used in the evaluation of Eucalyptus urophylla x Eucalyptus grandis seedlings. $\mathrm{H}=$ height; $\mathrm{DC}=$ stem diameter; IQD = Dickson quality index; BST = total dry matter; $\mathrm{BSA}=$ shoot dry matter; BSR = root dry matter; NRS = nu mber of secondary roots.

\section{CONCLUSÕES}

Com base nas análises efetuadas no presente trabalho e nos resultados obtidos para a maioria das variáveis, pode-se concluir que para Eucalyptus urophylla x Eucalyptus grandis:

- mudas produzidas em tubetes de $55 \mathrm{~cm}^{3}$ podem permanecer em viveiro pe lo período de até 90 dias;

- mudas produzidas em tubetes de $100 \mathrm{~cm}^{3}$ podem permanecer em viveiro pelo período de até 150 dias;

- mudas produzidas em tubetes de $200 \mathrm{~cm}^{3}$ podem permanecer em viveiro por período superior a 210 dias. 


\section{REFERÊNCIAS}

BARROSO, D. G.; CARNEIRO, J. G. A.; LELES, P. S. S. Qualidade de mudas de Eucalyptus camaldulensis e E. urophylla produzidas em tubetes e em blocos prensados, com diferentes substratos. Floresta e Ambiente, Seropédica, v. 7, n. 1, p. 238 - 250, 2000.

CALDEIRA, M. V. W.; BLUM, H.; BALBINOT, R.; LOMBARDI, K. C. Uso do resíduo do algodão no substrato para produção de mudas florestais. Revista Acadêmica: Ciências Agrárias e Ambientais, Curitiba, v. 6, p. 191 - 202, 2008a.

CALDEIRA, M. V. W.; ROSA, G. N.; FENILLI, T. A. B.; HARBS, R. M. P. Composto orgânico na produção de mudas de aroeira-vermelha. Scientia Agraria, Piracicaba, v. 9, p. 27 - 33, 2008b.

CALDEIRA, M. V. W.; MARCOLIN, M.; MORAES, E.; SCHAADT, S. S. Influência do resíduo da indústria do algodão na formulação de substrato para produção de mudas de Schinus terebinthifolius Raddi, Archontophoenix alexandrae Wendl. et Drude e Archontophoenix cunninghamiana Wendl. et Drude. Ambiência, Guarapuava, v. 3, p. 1 - 8, 2007.

CARNEIRO, J. G. de A. Produção e controle de qualidade de mudas florestais. Curitiba: UFPR/FUPEF; Campos: UENF, 1995. $451 \mathrm{p}$.

CARVALHO, A. M. Valorização da madeira do híbrido Eucalyptus grandis x Eucalyptus urophylla através da produção conjunta de madeira serrada em pequenas dimensões, celulose e lenha. $128 \mathrm{f}$. Dissertação (Mestrado em Engenharia Florestal) - Escola Superior de Agricultura "Luiz de Queiroz", Universidade de São Paulo, Piracicaba, 2000.

CUNHA, A. O.; ANDRADE, L. A. de; BRUNO, R. L. A.; SILVA, J. A. L. da; SOUZA, V. C. de. Efeitos de substratos e das dimensões de recipientes na qualidade de mudas de Tabebuia impetiginosa (Mart. ex DC.) Standl. Revista Ár vore, Viçosa, v. 29, n. 4, p. 507 - 516, 2005.

ELOY, E.; CARON, B. O.; SCHMIDT, D.; BEHLING, A.; SCHWERS, L.; ELLI, E. F. Avaliação da qualidade de mudas de Eucalyptus grandis utilizando parâmetros morfológicos. Floresta, Curitiba, PR, v. 43, n. 3 , p. 373 $384,2013$.

FONSECA, E. P.; VALÉRI, S. V.; MIGLIORANZA, E.; FONSECA, N. A. N.; COUTO, L. Padrão de qualidade de mudas de Trema micranta (L.) Blume, produzidas sob diferentes períodos de sombreamento. Revista Árvore, Viçosa, v. 26, n. 4, 2002.

FreitAS, T. A. S.; BARRoso, D. G.; SIlvA, L. S.; CARNEIRO, J. G. A.; PAUlO, G. M. Mudas de eucalipto produzidas a partir de miniestacas em diferentes recipientes e substratos. Revista Ár vore, Viçosa, MG, v. 30, n. 4, p. $519-528,2006$.

FREITAS, T. A. S. de; BARROSO, D. G.; CARNEIRO, J. G. de A.; PENCHEL, R. M.; LAMÔNICA, K. R.; FERREIRA, D. de A. Desempenho radicular de mudas de eucalipto produzidas em diferentes recipientes e substratos. Re vista Árvore, Viçosa, v. 29, n. 6, p. 853 - 861, 2005.

FREITAS, T. A. S. de; FONSECA, M. D. S.; SOUZA, S. S. M. de; LIMA, T. M.; MENDONÇA, A. V. R.; SANTOS, A. P. dos. Crescimento e ciclo de produção de mudas de Eucalyptus em recipientes. Pesquisa Florestal Brasileira, Colo mbo, v. 33, n. 76, p. 419 - 428, 2013.

GOMES, D. R.; CALDEIRA, M. V. W.; DELARMELINA, W. M.; GONÇALVES, E. O.; TRAZZI, P. A. Lodo de esgoto como substrato para a produção de mudas de Tectona grandis L. Cerne, Lavras, v. 19, n. 1, p. 123 $131,2013$.

JOSE, A. C. C.; DA VIDE, A. C.; OLIVEIRA, S. L. de. Produção de mudas de aroeira (Schinus terebinthifolius Raddi) para recuperação de áreas degradadas pela mineração de bau xita. Cerne, Lavras, v. 11, n. 2, p. 187 - 196 , 2005 .

KNAPIK, G. J. Utilização do pó de basal to como alternativa à adubação convencional na produção de mudas de Mimosa scabrella Benth e Prunus sellowii Koehne. 2005. 163 p. Dissertação (Mestrado em Engenharia Florestal) - Universidade Federal do Paraná, Curitiba, 2005.

MALA VASI, U. C.; MALA VASI, M. de M. Efeito do volume do tubete no crescimento inicial de plântulas de Cordia trichotoma (Vell.) Arrab. ex Steud e Jacaranda micranta Cham. Ciência Florestal, Santa Maria, v. 16,

FLOREST A, Curitiba, PR, v. 46, n. 1, p. 39 - 46, jan. / mar. 2016.

Schorn, L. A. et al.

ISSN eletrônico 1982-4688

DOI: $10.5380 /$ rf.v46il 38907 
n. 1 , p. $11-16,2006$.

MATTEI, V. L. Comparação entre semeadura direta e plantio de mudas produzidas em tubetes, na implantação de povoamento de Pinus taeda L. 1993. 149 f. Tese (Doutorado) - Universidade Federal do Paraná, Curitiba, 1993.

MONTANARI, R.; MARQUES JUNIOR, J.; CAMPOS, M. C. C.; CA VALCANTE, I. H. L. Níveis de resíduos de metalurgia em substratos na formação de mudas de eucalipto (Eucalyptus urograndis). Re vista de Biologia e Ciências da Terra, v. 7, p. 59 - 66, 2007.

NEVES, C. S. V. J.; MEDINA, C. de C.; AZEVEDO, M. C. B. de; HIGA, A. R.; SIMON, A. Efeitos de substratos e recipientes utilizados na produção das mudas sobre a arquitetura do sistema radicular de árvores de acácia-negra. Revista Árvore, Viçosa, v. 29, n. 6, p. 897 - 905, 2005.

PARVIAINEN, J. V. Future trends for containerized tree seedlings production: a literature review. Silva Fennica, v. 24, n. 1, p. 93-103, 1990.

REIS, G. G.; REIS, M. G. F.; MAESTRI, M. Crescimento de Eucalyptus camaldulensis, E. grandis e E. cloeziana sob diferentes níveis de restrição radicular. Re vista Ár vore, Viçosa, v. 13, n. 1, p. 1 - 18, 1989.

SAIDELlES, F. L. F.; CALDEIRA, M. V. W.; SCHIRMER, W. N.; SPERANDIO, H. V. Casca de arroz carbonizada como substrato para produção de mudas de tamboril-da-mata e garapeira. Semina: Ciências Agrárias, v. 30, p. 1173 - 1186, 2009.

SANTOS, G. F. Vale do Garcia (Blumenau - SC): Análise climatogeomorfológica e a repercussão dos episódios pluviais no espaço urbano. São Paulo: 362 p. Tese (Doutorado em Geologia) - Universidade de São Paulo, 1996.

TRAZZI, P. A.; CALDEIRA, M. V. W.; COLOMBI, R. Avaliação de mudas de Tecoma stans utilizando biossólido e resíduo orgânico. Revista de Agricultura, Piracicaba, v. 85, p. 218 - 226, 2010. 